\title{
Immediate effect of Masai Barefoot Technology shoes on knee joint moments in women with knee osteoarthritis.
}

\section{$\operatorname{AUTHOR}(\mathrm{S})$ :}

Tateuchi, Hiroshige; Taniguchi, Masashi; Takagi, Yui; Goto, Yusuke; Otsuka, Naoki; Koyama, Yumiko; Kobayashi, Masashi; Ichihashi, Noriaki

\section{CITATION:}

Tateuchi, Hiroshige ... [et al]. Immediate effect of Masai Barefoot Technology shoes on knee joint moments in women with knee osteoarthritis.. Gait \& posture 2014, 40(1): 204208

\section{ISSUE DATE:}

2014-05

URL:

http://hdl.handle.net/2433/187805

\section{RIGHT:}

(C) 2014 Elsevier B.V.; This is not the published version. Please cite only the published version.; この論文は出版社版でありません。引用の際に は出版社版をご確認ご利用ください。 


\section{Title:}

Immediate effect of Masai Barefoot Technology shoes on knee joint moments in women with knee osteoarthritis

\section{The name(s) of the author(s):}

Hiroshige Tateuchi ${ }^{\text {a, }}$, PhD; Masashi Taniguchi ${ }^{\mathrm{b}}$, MS; Yui Takagi ${ }^{\text {c }}$, MS; Yusuke Goto ${ }^{\mathrm{d}}$, BS; Naoki Otsuka $^{\text {a }}$, MS; Yumiko Koyama ${ }^{\text {a }}$, MS; Masashi Kobayashi ${ }^{\text {e }}$, MD, PhD; Noriaki Ichihashi ${ }^{\text {a }}$ PhD

The affiliation(s) of the author(s):

a. Human Health Sciences, Graduate School of Medicine, Kyoto University, Kyoto, Japan

b. Shiga University of Medical Science Hospital, Shiga, Japan

c. Nagoya University Hospital, Aichi, Japan

d. Uji Takeda Hospital, Kyoto, Japan

e. Kobayashi Orthopaedic Clinic, Kyoto, Japan 


\begin{abstract}
Footwear modification can beneficially alter knee loading in patients with knee osteoarthritis. This study evaluated the effect of Masai Barefoot Technology shoes on reductions in external knee moments in patients with knee osteoarthritis. Three-dimensional motion analysis was used to examine the effect of Masai Barefoot Technology versus control shoes on the knee adduction and flexion moments in 17 women (mean age, 63.6 years) with radiographically confirmed knee osteoarthritis. The lateral and anterior trunk lean values, knee flexion and adduction angles, and ground reaction force were also evaluated. The influence of the original walking pattern on the changes in knee moments with Masai Barefoot Technology shoes was evaluated. The knee flexion moment in early stance was significantly reduced while walking with the Masai Barefoot Technology shoes $(0.25 \pm 0.14 \mathrm{~N} \cdot \mathrm{m} / \mathrm{kg} \cdot \mathrm{m})$ as compared with walking with control shoes $(0.30 \pm 0.19 \mathrm{~N} \cdot \mathrm{m} / \mathrm{kg} \cdot \mathrm{m})$; whereas the knee adduction moment showed no changes. Masai Barefoot Technology shoes did not increase compensatory lateral and anterior trunk lean. The degree of knee flexion moment in the original walking pattern with control shoes was correlated directly with its reduction when wearing Masai Barefoot Technology shoes by multiple linear regression analysis (adjusted $R^{2}=0.44, P<$ 0.01). Masai Barefoot Technology shoes reduced the knee flexion moment during walking without increasing the compensatory trunk lean and may therefore reduce external knee loading in women with knee osteoarthritis.
\end{abstract}




\section{Introduction}

The rate of progression of osteoarthritis $(\mathrm{OA})$ at the knee is associated with increased loads in the joint during ambulation [1]. Footwear modification for patients with knee OA has received attention as an effective conservative intervention that can alter knee load [2]. A series of related previous studies have shown that variable-stiffness shoes [3], mobility shoes [4], flat walking shoes [5], flexible non-heeled shoes [6], and shoes with lateral wedging and a variable-stiffness sole [7] can reduce knee joint loading while walking as compared to modern heeled shoes or stability shoes. These previous studies have focused on the changes in the external knee adduction moment (KAM), however, Walter et al. [8] confirmed that decreased KAM does not necessarily guarantee decreased medial compartment contact force of the knee during gait. In their report, regression analysis demonstrated that the peak value of medial contact force was best fitted by a combination of peak value of the KAM and external knee flexion moment (KFM). Therefore, an evaluation of gait modifications for reducing the medial contact force should consider both KAM and KFM. However, with the exception of a report by Bennell et al. [7], KAM and KFM while wearing modified shoes have not been simultaneously analyzed in patients with knee OA.

Thus far, increased lateral and anterior trunk lean is one of the most effective gait modifications for reducing knee loading during walking [9]. Increased lateral trunk lean toward the symptomatic knee and increased anterior trunk lean have been shown to reduce the KAM and KFM, respectively, in patients with knee OA and those who have undergone total knee arthroplasty [10,11]. To determine the exact effects of shoe modifications on knee moments, it is necessary to examine changes in the trunk lean during walking. However, no studies have examined gait modifications along with the changes in trunk motion that accompany changes in shoe types.

Masai Barefoot Technology (MBT) shoes (Masai Marketing \& Trading AG, Winterthur, Switzerland) are a type of shoes specialized in altering the biomechanics of the lower extremity during standing and walking. MBT shoes are characterized by a round sole in the anteroposterior direction and a flexible heel. By using MBT shoes, a decreased range of motion in the hip and the knee joints, which were assumed to be due to decreased walking speed and stride length, along with increased ankle dorsiflexion at initial contact have been reported [12]. Meanwhile, at the same walking speed, the kinematics and angular impulse for all lower extremity joints were noted to be similar in MBT and control shoe conditions, except for increased ankle dorsiflexion during the first half of stance [13]. Our previous study showed that ground reaction force decreased at the shock absorption and progression phases when wearing MBT shoes [14]. Moreover, for the knee joint, reduced peak KAM and KFM during early stance and increased peak KFM in late stance were observed when walking with MBT shoes in young and the elderly individuals [15]. Reduced peak KAM in early stance was also demonstrated in overweight individuals who were free from knee pain [16]. For both one-leg standing and walking, trunk lean in both the frontal and sagittal planes tended to increase with a foam support surface as compared to that with a normal floor [17]. Therefore, 
lateral and anteroposterior trunk lean should be measured when walking with MBT shoes, which have a flexible heel coupled with a round sole.

It is possible that MBT shoes may have a beneficial effect on knee loading for patients with knee OA. However, to date, the effects of MBT shoes on gait biomechanics in patients with knee OA have not been investigated. The purpose of the current study was therefore to evaluate the effect of MBT shoes on reductions in external knee moments in women with knee OA. We hypothesized that external knee moments during walking would be reduced in women with knee OA by wearing MBT shoes without increasing the lateral or anterior trunk lean adopted as gait compensation.

\section{Methods}

\subsection{Subjects}

This study enrolled 17 women diagnosed with OA in the medial compartment of the knee by a single orthopedic surgeon. Participants were recruited from the local orthopedic clinic. Patients were excluded from the study if they had any musculoskeletal conditions other than knee OA, if they were unable to walk without assistance, or if they were diagnosed with any neurological disorders that limited their function. Further, patients with knee flexion contracture of $>15^{\circ}$ were excluded because this condition was expected to lead to mechanical overloads at the knee [18]. Elderly individuals aged 80 years or above were also excluded due to the increased risk of falls [19]. Although many patients had bilateral knee OA, only the most symptomatic knee of each patient was analyzed.

Knee OA were graded radiographically using the Kellgren/Lawrence (K/L) classification. Further, the disease-specific scale of the Japanese Knee Osteoarthritis Measure (JKOM) was used. The JKOM is a self-administered measure consisting of 25 items, which include subjective pain in level walking, standing, or climbing stairs as well as physical functions related to the activities of daily living and social functions [20]. The possible score for the JKOM is 100 points, and low scores indicate good function. The participants' demographic characteristics are presented in Table 1. All participants provided informed consent, and the protocol for this study was approved by the Institutional Ethics Committee.

\subsection{Test protocol}

The study protocol consisted of three-dimensional gait analysis under two conditions with different shoes: walking with MBT shoes and walking with control shoes (Fig. 1). The control shoes were of a type that is flexible with a flat sole, which is widely used in rehabilitation facilities (Ayumi; Tokutake Sangyo Co., Ltd., Japan). The participants were allowed walk on the walkway at least 5 times to familiarize themselves with the environment before the actual trials. The first recording was performed with walking when wearing the control shoes. The participants were instructed to walk at two speeds, i.e., self-selected and slow. Participants were then given 
instructions for walking with MBT shoes by an instructor of MBT shoes. Participants completed approximately $20 \mathrm{~min}$ of walking in the laboratory until the instructor felt that they were walking appropriately and the participants felt comfortable with the MBT shoes. The duration of practice was determined by reference to previous studies that investigated biomechanics during walking with MBT shoes [13,14,21], and by considering the physical load on the patients. Appropriate walking with MBT shoes was defined as a successful shift in body weight with rolling movements over the rounded sole. After participants became accustomed to walking with MBT shoes, the second recording was performed. The MBT shoes condition was measured at self-selected speed. To avoid the after effects of walking in MBT shoes, the control condition of the study took place before the MBT shoes condition. At least three successful trials for control shoes and five successful trials for MBT shoes were recorded for subsequent analysis, since a higher variability in kinetic variables while walking with MBT shoes [22].

\subsection{Gait analyses}

Body kinematics were recorded using a 7-camera Vicon motion system (Vicon Nexus; Vicon Motion Systems Ltd., Oxford, England) at a sampling rate of $200 \mathrm{~Hz}$. The reflective markers were attached to the body of each participant according to the Vicon Plug-in-Gait marker placement protocol (full body). The trunk segment contained six markers: at the 7th cervical and 10th thoracic vertebrae, jugular notch, xiphoid process of the sternum, and left and right acromioclavicular joints. Sixteen other markers were placed bilaterally on the anterior superior iliac spine, posterior superior iliac spine, lateral thigh, lateral femoral epicondyle, lateral shank, lateral malleolus, second metatarsal head, and calcaneus. Four force plates (Kistler Japan Co., Ltd., Tokyo, Japan) were used to measure the ground reaction force (GRF) at a sampling rate of $1000 \mathrm{~Hz}$. Kinematic and kinetic data were both low-pass filtered using 4th order Butterworth filter at $6 \mathrm{~Hz}$ and $20 \mathrm{~Hz}$, respectively.

Vicon Clinical Manager software was used to calculate the gait variables. The primary variables were the knee moments: peak of knee flexion moment in early stance (KFM1) and late stance (KFM2), knee extension moment in mid stance (KEM), knee adduction moment in early stance (KAM1) and late stance (KAM2), and impulse of knee adduction moment (KAMimp). Additionally, the following gait variables were measured as secondary variables: walking speed, stride length, peak of lateral and anterior trunk lean in the global frame, peak of knee flexion angle and knee adduction angle in early stance, peak of vertical GRF in early stance and late stance, peak of anteroposterior GRF in early stance and late stance, and peak of medial GRF. GRF values were normalized by body weight (in N/kg), and joint moments were expressed as external joint moments and were normalized according to body weight and height (in $\mathrm{N} \cdot \mathrm{m} / \mathrm{kg} \cdot \mathrm{m}$ ).

\subsection{Statistical analysis}

The SPSS 17.0 statistical analysis package (SPSS Inc.) was used for all statistical analyses. All variables were assessed for normality using Shapiro-Wilk statistic and graphical methods, prior to 
statistical analysis. The gait variables were tested using the paired $t$-test or Wilcoxon rank sum test, as required, to detect any significant differences in the dependent variables between the 2 conditions. If the self-selected speeds while wearing the control and MBT shoes were significantly different, the walking speed (from among the self-selected speed and slow speed) that was closest to the walking speed when wearing the MBT shoes was chosen for each patient for comparison of the gait variables. Furthermore, when significant reduction in knee moments was identified in walking with MBT shoes, the influence of the original walking pattern on the changes in knee moments was evaluated by using stepwise multiple linear regression analysis with amount of changes in knee moments as the dependent variable and walking speed, knee angle, and knee moment with control shoes as the independent variables. The level of statistical significance was set at 0.05 .

\section{Results}

All gait variables, except for knee moments, while walking with control shoes and MBT shoes are shown in Table 2. The self-selected speeds while wearing the 2 types of shoes differed significantly. After adjustment for walking speed, no significant differences were noted in the walking speed or stride length. Consequently, we could compare the kinematic and kinetic data between the 2 conditions.

The knee moments and moment impulse are shown in Table 3. KAM1, KAM2, KAMimp, KEM, or KFM2 did not differ statistically while wearing the 2 types of shoes. However, KFM1 showed a significant reduction of $16.7 \%$ while wearing the MBT shoes as compared to that with control shoes. On the other hand, there was a slight but significant increase in trunk lean toward the extension direction while wearing the MBT shoes as compared to that with the control shoes. Lateral trunk lean did not differ between the 2 conditions. No significant differences were noted in the knee flexion and knee adduction angles. In addition, the medial GRF tended to be higher while wearing the MBT shoes; however, this difference was not statistically significant. The vertical and anteroposterior GRF did not differ between the 2 conditions.

In multiple regression analysis, KFM1 with control shoes was taken as an independent variable for reduced KFM1 with MBT shoes (adjusted $R^{2}=0.44, P<0.01$ ). In simple terms, the larger the KFM1 in the original walking pattern with the control shoes, the greater was the degree of KFM1 reduction on wearing the MBT shoes.

\section{Discussion}

The primary finding of the current study was that external KFM in early stance was reduced while walking with MBT shoes without increasing the lateral and anterior trunk lean adopted as gait 
compensation, although KAM and KAMimp remained unchanged. Therefore, our primary hypothesis was reasonably well supported.

Changes in joint moment are produced following changes in GRF or the lever arm between GRF and the center of the joint. In the current study, no difference was noted in GRF and knee angle between the 2 conditions of wearing the MBT and control shoes. Therefore, the reduced KFM in early stance that was observed while walking with MBT shoes may be likely to be due to a reduction in the lever arm subsequent to an altered location of the GRF relative to the center of the knee joint in the sagittal plane. The round sole and flexible heel of MBT shoes are their most distinctive feature; this configuration would result in ground contact of the midfoot along with the heel at initial contact, which might distribute the pressure from the heel to the midfoot, and consequently induce an anterior shift of the GRF (i.e., close to center of the knee joint) in early stance. Furthermore, as mentioned above, a large KFM in the original walking pattern was associated with a greater reduction in KFM when using MBT shoes. These findings provide clinicians with relevant information that will enable the appropriate selection of the gait pattern that will benefit from using MBT shoes.

The medial contact force of the knee joint is determined by the combined effect of KAM and KFM [8]. Therefore, reduced KFM while wearing MBT shoes would be partly related to a reduction in the external load on the knee joint. It is reported that patients with knee OA exhibit smaller KFM as compared with healthy individuals; however, these differences have been interpreted as compensatory mechanisms to reduce knee loading [23,24]. Patients with knee OA need to increase anterior trunk lean and reduce knee flexion in the early stance for compensation, which can contribute to shortening the lever arm [10,23]; this indicates a disruption of the shock-absorbing mechanism at the knee joint. Walking with MBT shoes can potentially reduce the KFM, eliminating the need to adopt compensatory gait pattern. Furthermore, reduced KFM would also contribute to a reduction in the load on the knee extensor mechanism, which comprises the quadriceps and its tendon, patella, and patella tendon. The patellofemoral joint is the compartment that is most commonly affected in knee OA [25]. Considering these factors, MBT shoes appear to be a potentially effective treatment possibility in patients with knee OA coexisting with patellofemoral OA.

We found no differences in the KAM and KAM impulse between the walking conditions with control shoes and MBT shoes. Previous study has shown effective CoP shift by using a sole with a greater lateral stiffness on reduction of KAM during walking [3]. The addition of a device that alters the mediolateral CoP location may therefore be needed in MBT shoes.

Lateral and anterior trunk lean reduce the KAM and KFM, respectively, by altering the direction of GRF in response to the changed location of the center of mass of the trunk, consequently reducing the knee moment lever arm [9]. Therefore, trunk lean has been recommended as a gait retraining strategy in order to reduce knee loading [11,26]; however, it has been reported that $33 \%$ of subjects experience lower back discomfort during walking trials with altered trunk lean [27]. Indeed, the majority of patients with knee OA $(57.4 \%)$ report the presence of low back pain [28]. Although the 
relationship between gait pattern and low back pain in patients with knee OA remains unclear, gait modification including trunk lean might involve a certain degree of risk for spinal overload. In this regard, walking with MBT shoes might be a relatively favorable method for patients with knee OA who are forced to increase the anterior trunk lean as compensation.

Our study has certain limitations. Although reducing the load presumably exerted a beneficial influence on patients with knee OA [29], the changes in the symptoms resulting from gait modifications were not measured. Another limitation is that our study enrolled only women younger than 80 years. Additional investigations that include male subjects may be necessary. However, the prevalence of knee OA is known to be higher in females than in males [30], and female gender is the most important factor associated with the incidence of knee OA [31]. The lack of randomization of test conditions was also a limitation. However, it was aimed at avoiding the after effects of wearing MBT shoes in order to effectively compare both conditions. A final limitation is that the present study investigated only the immediate effects of MBT shoes. Long term use of MBT shoes is known to lead to reductions in movement variability as an adaptation to the lower stability of the shoe [22]. Moreover, pain reduction has been demonstrated in patients with knee OA by walking with MBT shoes for 12 weeks, although no significant difference was observed between MBT shoes and control shoes [32]. In future studies, alterations in gait biomechanics induced by the long-term use of MBT shoes by patients with knee OA should be investigated using not only normal floors but also various floors such as ramps and uneven surfaces along with their clinical benefits and the subsequent kinematic and kinetic changes.

\section{Conclusions}

Walking with MBT shoes had the immediate effect of reducing the KFM in early stance without increasing compensatory trunk lean in women with knee OA, whereas no changes were observed in the KAM. The present findings introduce the possibility of using MBT shoes for reducing external knee loading and ameliorating pain in the patients with knee OA. We consider that in patients with higher KFM, MBT shoes have the potential to be an effective conservative intervention.

\section{Acknowledgements}

We would like to thank Yoji Hazama and Takashi Ohbu at Ever New Inc. for their assistance with MBT training.

\section{Conflict of interest statement}


None of the authors have any conflicts of interest associated with this study.

\section{References}

[1] Andriacchi TP, Mündermann A. The role of ambulatory mechanics in the initiation and progression of knee osteoarthritis. Curr Opin Rheumatol 2006; 18: 514-518.

[2] Radzimski AO, Mündermann A, Sole G. Effect of footwear on the external knee adduction moment. A systematic review. Knee 2012; 19: 163-175.

[3] Erhart JC, Mündermann A, Elspas B, Giori NJ, Andriacchi TP. A variable-stiffness shoe lowers the knee adduction moment in subjects with symptoms of medial compartment knee osteoarthritis. J Biomech 2008; 41: 2720-2725.

[4] Shakoor N, Lidtke R.H, Sengupta M, Fogg LF, Block JA. Effects of specialized footwear on joint loads in osteoarthritis of the knee. Arthritis Rheum 2008; 59: 214-220.

[5] Shakoor N, Sengupta M, Foucher KC, Wimmer MA, Fogg LF, Block JA. Effects of common footwear on joint loading in osteoarthritis of the knee. Arthritis Care Res 2010; 62: 917-923.

[6] Trombini-Souza F, Kimura A, Ribeiro AP, Butugan M, Akashi P, Pássaro AC, Arnone AC, Sacco IC. Inexpensive footwear decreases joint loading in elderly women with knee osteoarthritis. Gait Posture 2011; 34: 126-130.

[7] Bennell KL, Kean CO, Wrigley TV, Hinman RS, Effects of a modified shoe on knee load in people with and without knee osteoarthritis. Arthritis Rheum 2013; 65: 701-709.

[8] Walter JP, D’Lima DD, Colwell Jr CW, Fregly BJ. Decreased knee adduction moment does not guarantee decreased medial contact force during gait. J Orthop Res 2010; 28:1348-1354.

[9] Simic M, Hinman RS, Wrigley TV, Bennell KL, Hunt MA. Gait modification strategies for altering medial knee joint load: A systematic review. Arthritis Care Res 2011; 63: 405-426.

[10]Li K, Ackland DC, McClelland JA, Webster KE, Feller JA, Steiger R, Pandy MG. Trunk muscle action compensates for reduced quadriceps force during walking after total knee arthroplasty. Gait Posture 2012; 38: 79-85.

[11]Simic M, Hunt MA, Bennell KL, Hinman RS, Wrigley TV. Trunk lean gait modification and knee joint load in people with medial knee osteoarthritis: The effect of varying trunk lean angles. Arthritis Care Res 2012; 64: 1545-1553.

[12]Romkes J, Rudmann C, Brunner R. Changes in gait and EMG when walking with the Masai Barefoot Technique. Clin Biomech 2006; 21: 75-81.

[13]Nigg B, Hintzen S, Ferber R. Effect of an unstable shoe construction on lower extremity gait characteristics. Clin Biomech 2006a; 21: 82-88.

[14] Taniguchi M, Tateuchi H, Takeoka T, Ichihashi N. Kinematic and kinetic characteristics of Masai Barefoot Technology footwear. Gait Posture 2012; 25: 567-572.

[15]Buchecker M, Lindinger S, Pfusterschmied J, Müller E. Effects of age on lower extremity joint kinematics and kinetics during level walking with Masai barefoot technology shoes. Eur J Phys 
Rehabil Med 2012a; 48: 1-12.

[16]Buchecker M, Wagner H, Pfusterschmied J, Stöggl TL, Müller E. Lower extremity joint loading during level walking with Masai barefoot technology shoes in overweight males. Scand J Med Sci Sports 2012b; 22: 372-380.

[17]Gill J, Allum JHJ, Carpenter MG, Held-Zioikowska M, Adkin AL, Honegger F, Pierchala K. Trunk sway measures of postural stability during clinical balance tests: Effects of age. J Gerontal A Biol Sci Med Sci 2001; 56: M438-447.

[18]Harato K, Nagura T, Matsumoto H, Otani T, Toyama Y, Suda Y. Knee flexion contracture will lead to mechanical overload in both limbs: A simulation study using gait analysis. Knee 2008; 15: 467-472.

[19]Am Geriatr Soc, British Gereatr Soc, Am Acad Orthop Surg. Panel on falls prevention. Guideline for the prevention of falls in older persons. J Am Geriatr Soc 2001; 49: 664-672.

[20]Akai M, Doi T, Fujino K, Iwaya T, Kurosawa H, Nasu T. An outcome measure for Japanese people with knee osteoarthritis. J Rheumatol 2005; 32: 1524-1532.

[21] Roberts S, Birch I, Otter S. Comparison of ankle and subtalar joint complex range of motion during barefoot walking and walking in Masai Barefoot Technology sandals. J Foot Ankle Res 2011; 4: 1 .

[22] Stöggl T, Haudum A, Birklbauer J, Murrer M, Müller E. Short and long term adaptation of variability during walking using unstable (Mbt) shoes. Clin Biomech 2010; 25: 816-822.

[23]Huang SC, Wei IP, Chien HL, Wang TM, Liu YH, Chen HL, Lu TW, Lin JG. Effects of severity of degeneration on gait patterns in patients with medial knee osteoarthritis. Med Eng Phys 2008; 30: 997-1003.

[24]Kaufman KR, Hughes C, Morrey BF, Morrey M, An KN. Gait characteristics of patients with knee osteoarthritis. J Biomech 2001; 34: 907-915.

[25]Kumm J, Tamm A, Lintrop M, Tamm A. The prevalence and progression of radiographic knee osteoarthritis over 6 years in a population-based cohort of middle-aged subjects. Rheumatol Int 2012; 32: 3545-3550.

[26] Mündermann A, Asay JL, Mündermann L, Andriacchi TP. Implications of increased medio-lateral trunk sway for ambulatory mechanics. J Biomech 2008; 41: 165-170.

[27]Hunt MA, Simic M, Hinman RS, Bennell KL, Wrigley TV. Feasibility of a gait retraining strategy for reducing knee joint loading: Increased trunk lean guided by real-time biofeedback. $\mathrm{J}$ Biomech 2011; 44: 943-947.

[28] Suri P, Morgenroth DC, Kwoh CK, Bean JF, Kalichman L, Hunter DJ. Low back pain and other musculoskeletal pain comorbidities in individuals with symptomatic osteoarthritis of the knee: Data from the osteoarthritis initiative. Arthritis Care Res 2010; 62: 1715-1723.

[29]Thorp LE, Sumner DR, Wimmer MA, Block JA. Relationship between pain and medial knee joint loading in mild radiographic knee osteoarthritis. Arthritis Rheum 2007; 57: 1254-1260.

[30] Haq SA, Davatchi F. Osteoarthritis of the knee in the COPCORD world. Int J Rheum Dis 2011; 
14: $122-129$.

[31]Nishimura A, Hasegawa M, Kato K, Yamada T, Uchida A, Sudo A. Risk factors for the incidence and progression of radiographic osteoarthritis of the knee among Japanese. Int Orthop 2011; 35: 839-843.

[32]Nigg BM, Emery C, Hiemstra LA. Unstable shoe construction and reduction of pain in osteoarthritis patients. Med Sci Sports Exerc 2006b; 38: 1701-1708. 
Table 1. Demographic characteristics of the participants

\begin{tabular}{lrcc}
\hline & \multicolumn{2}{c}{ Mean (SD) } & Range \\
\hline Age (years) & 63.6 & $(7.9)$ & $48-72$ \\
Weight $(\mathrm{kg})$ & 56.5 & $(6.5)$ & $50.4-71.4$ \\
Height $(\mathrm{cm})$ & 156.7 & $(5.7)$ & $146.2-167.5$ \\
Body mass index $\left(\mathrm{kg} / \mathrm{m}^{2}\right)$ & 23.0 & $(2.6)$ & $20.2-29.1$ \\
KL Grade & & & \\
Grade 1/2/3/4 (n) & $1 / 9 / 2 / 5$ & - \\
JKOM score* & 21.7 & $(8.7)$ & $9-47$ \\
\hline
\end{tabular}

(Foot-notes for Table 1)

$\mathrm{KL}=$ Kellgren/Lawrence; JKOM = Japanese Knee Osteoarthritis Measure

* The total possible score is 100 points, and low scores indicate good function. 
Table 2. Changes in the secondary gait variables while walking with control shoes and MBT shoes

\begin{tabular}{|c|c|c|c|c|c|}
\hline & \multirow{2}{*}{\multicolumn{2}{|c|}{$\frac{\text { Control shoes }}{\text { Mean (SD) }}$}} & \multirow{2}{*}{\multicolumn{2}{|c|}{$\frac{\text { MBT shoes }}{\text { Mean (SD) }}$}} & \multirow{2}{*}{$p$-value $\dagger$} \\
\hline & & & & & \\
\hline Self-selected & 1.26 & $(0.15)$ & 1.14 & $(0.14)$ & $<0.001$ \\
\hline After adjustment* & 1.17 & $(0.15)$ & 1.14 & $(0.14)$ & 0.153 \\
\hline \multicolumn{6}{|l|}{ Stride length (m) } \\
\hline Self-selected & 1.30 & $(0.11)$ & 1.27 & $(0.09)$ & 0.058 \\
\hline After adjustment* & 1.28 & $(0.13)$ & 1.27 & $(0.09)$ & 0.629 \\
\hline \multicolumn{6}{|l|}{ Trunk lean $\left(^{\circ}\right)$} \\
\hline Lateral lean & 1.2 & $(2.6)$ & 1.2 & $(2.7)$ & 0.731 \\
\hline Anterior lean & -0.1 & $(4.7)$ & -1.6 & $(4.3)$ & $<0.001$ \\
\hline \multicolumn{6}{|l|}{ Knee angle $\left(^{\circ}\right)$} \\
\hline Flexion & 19.7 & (7.6) & 19.4 & (7.1) & 0.906 \\
\hline Adduction & 7.2 & $(6.2)$ & 7.1 & $(5.4)$ & 0.777 \\
\hline \multicolumn{6}{|c|}{ Ground reaction force $(\mathrm{N} / \mathrm{kg})$} \\
\hline Vertical in early stance & 11.37 & $(0.86)$ & 11.23 & $(0.89)$ & 0.348 \\
\hline Vertical in late stance & 10.79 & $(0.58)$ & 10.64 & $(0.76)$ & 0.342 \\
\hline Posterior in early stance & 1.96 & $(0.29)$ & 2.01 & $(0.33)$ & 0.514 \\
\hline Anterior in late stance & 1.95 & $(0.37)$ & 1.93 & $(0.35)$ & 0.806 \\
\hline Medial & 0.51 & $(0.11)$ & 0.57 & $(0.10)$ & 0.053 \\
\hline
\end{tabular}

\section{(Foot-notes for Table 2)}

* The walking speed close to that of the MBT shoes condition was chosen from self-selected and slow speeds in the control shoes condition.

$\dagger P$ values are based on paired $t$-tests, except for the knee flex angle that was tested using the Wilcoxon rank sum test. 
Table 3. Changes in the knee moments while walking with control shoes and MBT shoes

\begin{tabular}{|c|c|c|c|c|c|}
\hline \multirow[b]{3}{*}{ KAM1 $(\mathrm{N} \cdot \mathrm{m} / \mathrm{kg} \cdot \mathrm{m})$} & \multirow{2}{*}{\multicolumn{2}{|c|}{$\frac{\text { Control shoes }}{\text { Mean (SD) }}$}} & \multirow{2}{*}{\multicolumn{2}{|c|}{$\frac{\text { MBT shoes }}{\text { Mean (SD) }}$}} & \multirow{3}{*}{$\begin{array}{c}P \text {-value } \\
0.549\end{array}$} \\
\hline & & & & & \\
\hline & 0.45 & $(0.08)$ & 0.44 & $(0.09)$ & \\
\hline KAM2 $(\mathrm{N} \cdot \mathrm{m} / \mathrm{kg} \cdot \mathrm{m})$ & 0.33 & $(0.09)$ & 0.35 & $(0.08)$ & 0.056 \\
\hline $\operatorname{KAMimp}(\mathrm{N} \cdot \mathrm{m} \cdot \mathrm{s} / \mathrm{kg} \cdot \mathrm{m})$ & 0.17 & $(0.05)$ & 0.18 & $(0.05)$ & 0.075 \\
\hline $\mathrm{KFM} 1(\mathrm{~N} \cdot \mathrm{m} / \mathrm{kg} \cdot \mathrm{m})$ & 0.30 & $(0.19)$ & 0.25 & $(0.14)$ & 0.047 \\
\hline $\operatorname{KEM}(\mathrm{N} \cdot \mathrm{m} / \mathrm{kg} \cdot \mathrm{m})$ & 0.01 & $(0.08)$ & 0.01 & $(0.06)$ & 0.906 \\
\hline KFM2 $(\mathrm{N} \cdot \mathrm{m} / \mathrm{kg} \cdot \mathrm{m})$ & 0.23 & $(0.09)$ & 0.22 & $(0.10)$ & 0.367 \\
\hline
\end{tabular}

(Foot-notes for Table 3)

KAM1 = knee adduction moment in early stance; KAM2 = knee adduction moment in late stance; KAMimp = impulse of knee adduction moment; KFM1 = knee flexion moment in early stance; KEM = knee extension moment in mid stance; KFM2 = knee flexion moment in late stance.

$\dagger P$ values are based on paired $t$-tests, except for KAM2 that was tested using the Wilcoxon rank sum test. 
Figure captions:

Figure 1: Control shoes and Masai Barefoot Technology (MBT) shoes. 


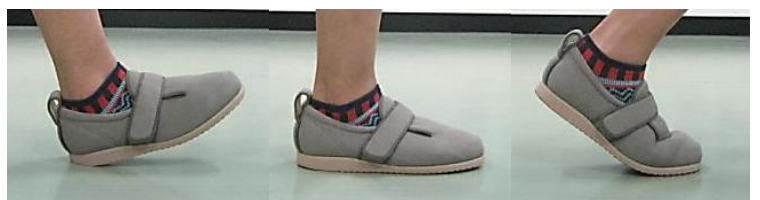

\section{Control shoes}

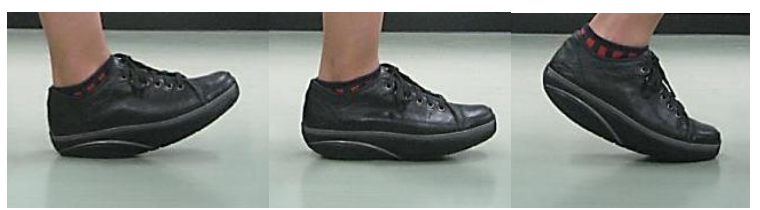

MBT shoes

Figure 1 